\title{
Cause of Death in Follicular Lymphoma in the First Decade of the Rituximab Era: A Pooled Analysis of French and US Cohorts
}

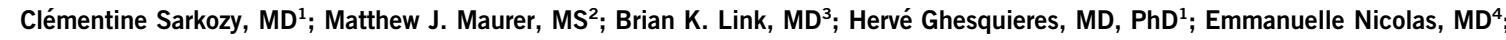 \\ Carrie A. Thompson, MD²; Alexandra Traverse-Glehen ${ }^{1}$; Andrew L. Feldman, MD² ${ }^{2}$ Cristine Allmer ${ }^{2}$; Susan L. Slager ${ }^{2}$; Stephen M. \\ Ansell, MD, PhD²; Thomas M. Habermann, MD²; Emmanuel Bachy ${ }^{1}$; James R. Cerhan, MD, PhD²; and Gilles Salles, MD, PhD ${ }^{1}$
}

PURPOSE Although the life expectancy of patients with follicular lymphoma (FL) has increased, little is known of their causes of death (CODs) in the rituximab era.

PATIENTS AND METHODS We pooled two cohorts of newly diagnosed patients with FL grade 1-3A. Patients were enrolled between 2001 and 2013 in two French referral institutions ( $N=734$; median follow-up 89 months) and 2002 and 2012 in the University of lowa and Mayo Clinic Specialized Program of Research Excellence (SPORE; $\mathrm{N}=920$; median follow-up 84 months). COD was classified as being a result of lymphoma, other malignancy, treatment related, or all other causes.

RESULTS Ten-year overall survival was comparable in the French $(80 \%)$ and US $(77 \%)$ cohorts. We were able to classify COD in 248 (88\%) of 283 decedents. In the overall cohort, lymphoma was the most common COD, with a cumulative incidence of $10.3 \%$ at 10 years, followed by treatment-related mortality (3.0\%), other malignancy $(2.9 \%)$, other causes $(2.2 \%)$, and unknown (3.0\%). The 10-year cumulative incidence of death as a result of lymphoma or treatment was higher than death as a result of all other causes for each age group (including patients $\geq 70$ years of age at diagnosis [25.4\% v 16.6\%]) Follicular Lymphoma International Prognostic Index score 3 to 5 (27.4\% v5.2\%), but not Follicular Lymphoma International Prognostic Index score 0 to 1 (4.0\% v3.7\%); for patients who failed to achieve event-free survival within 24 months from diagnosis (36.1\% v7.0\%), but not for patients who achieved event-free survival within 24 months of diagnosis (6.7\% v5.7\%); and for patients with a history of transformed FL ( $45.9 \% v 4.7 \%)$, but not among patients without (8.1\% v6.2\%). Overall, 77 of 140 deaths as a result of lymphoma occurred in patients whose FL transformed after diagnosis.

CONCLUSION Despite the improvement in overall survival in patients with FL in the rituximab era, their leading COD remains lymphoma, especially after disease transformation. Treatment-related mortality also represents a concern, which supports the need for less-toxic therapies.

J Clin Oncol 37:144-152. @ 2018 by American Society of Clinical Oncology

\section{INTRODUCTION}

Follicular lymphoma $(\mathrm{FL})$ is the most frequent indolent lymphoma. ${ }^{1,2}$ Despite recent progress in under-

ASSOCIATED CONTENT

Appendix

Data Supplement

Author affiliations

and support

information (if

applicable) appear

at the end of this

article.

Accepted on October

1,2018 and

published at jco.org

on November 27 ,

2018: DOI https://doi.

org/10.1200/JC0.18.

00400 standing the development of this disease, ${ }^{3,4}$ its clinical heterogeneity remains poorly understood. Some patients have an indolent evolution over several decades, whereas others show a rather aggressive clinical course, generally accompanied by a histologic transformation ${ }^{5}$ and poor prognosis. ${ }^{6,7}$ In the last two decades, life expectancy of patients with FL has markedly improved with the introduction of anti-CD2O therapies. ${ }^{8-12}$ For patients with symptomatic disease at diagnosis, standard of care includes immunochemotherapy (IC). ${ }^{13}$ In patients who are asymptomatic, treatment options are still debated. ${ }^{14,15}$ Despite these improvements, most physicians consider FL to be incurable, with a continuous pattern of relapse. ${ }^{16}$ Furthermore, patients with $\mathrm{FL}$ who are treated upfront with IC who are event free (no death, relapse, or retreatment) within 24 months of diagnosis (EFS24) have a subsequent survival that is comparable to the age- and sex-matched background population, ${ }^{17}$ whereas those who experience an event before 24 months have a more aggressive course with poor outcomes. ${ }^{17,18}$ In this context, long-term, treatmentrelated adverse effects and toxicity may become important issues.

In the rituximab era, deaths as a result of lymphoma in patients with $\mathrm{FL}$ have decreased at the population level, ${ }^{9}$ but there are limited data on the precise causes of death (CODs). For instance, when analyzing the longterm outcome of 281 patients with FL over different time periods, a significant improvement in cause-specific survival, but not in overall survival (OS), was found, ${ }^{19}$ which suggests that treatment-related toxicities may occur. Another report demonstrated that lymphoma 
and complications of treatments remained the leading COD in younger patients treated in a randomized trial ${ }^{12}$; however, it is important to note that survival is a function of lymphoma aggressiveness as well as of competing risks of mortality, which are lower for younger patients but are substantially increased with age. To provide a better characterization of CODs in the modern treatment era according to patient and disease characteristics, we conducted a pooled analysis of two independent cohorts of patients with FL from France and the United States.

\section{PATIENTS AND METHODS}

This study was conducted according to the Hospices Civils de Lyon, Centre Léon Bérard, University of lowa, and Mayo Clinic institutional ethics guidelines and in accordance with the Declaration of Helsinki. The French cohort consisted of 734 consecutive, newly diagnosed or referred patients with grade 1 to $3 \mathrm{~A} \mathrm{FL}$ who were treated at the Centre Hospitalier Lyon Sud and Centre Léon Bérard between 2001 and 2013. The US cohort consisted of 920 consecutive, newly diagnosed patients with grade 1 to $3 \mathrm{~A} \mathrm{FL}$ who consented and were enrolled between 2002 and 2012 in the University of Iowa and Mayo Clinic Specialized Program of Research Excellence (SPORE) Molecular Epidemiology Resource. ${ }^{20}$ In both cohorts, all pathology, including histologic transformations, were reviewed by study hematopathologists, and patient management, including staging, treatment(s), and clinical surveillance, was per the treating physician. Loss to follow-up was $0 \%$ in the French cohort and less than $1 \%$ in the SPORE cohort.

Disease progression, retreatment, transformation, and death events were verified via extensive review of medical records. COD was uniformly coded (by C.S., H.G., and G.S. for the French cohort; and C.A.T., T.M.H., and B.K.L. for the US cohort) as a result of lymphoma (progression or transformation), treatment, unrelated cancer, other causes, or unknown. Treatment-related mortality (TRM) was broadly defined and further classified into infection, cardiac, secondary myelodysplastic syndrome/acute myeloid leukemia (MDS/AML), or other. TRM was considered when death occurred either during treatment without evidence of disease refractoriness or shortly after treatment without evidence of progressive disease, or when death occurred much later but was unambiguously documented in the medical record as a result of treatment. If COD was unclear, the case was discussed between investigators and classified by consensus. FL transformation status was usually confirmed by biopsy; however, in some cases, diagnosis of transformation was based on previously described clinical criteria. ${ }^{5,21}$

Event-free survival (EFS) was defined as the time since diagnosis until progression, relapse, retreatment, or death as a result of any cause. On the basis of our prior publication, ${ }^{17}$ we defined early failure in patients treated with IC as EFS24 and for all other initial management strategies (including observation) as EFS at 12 months (EFS12). OS was defined as the time since the date of diagnosis to the date of death (any cause) or last known follow-up for patients still alive. COD was first categorized into the five major groupings (lymphoma, treatment, unrelated cancer, other causes, or unknown) and subsequently collapsed into three groupings: lymphoma-related deaths, defined as lymphoma or treatment related; deaths unrelated to lymphoma, which included other malignancies and other causes; and unknown. All-cause OS was determined via Kaplan-Meier method. Cumulative incidence for the competing risks of $\mathrm{COD}$ and tests of equality for $\mathrm{COD}$ between groups were calculated using the cuminc function from the cmprsk package in R. ${ }^{22}$ For early event analysis, time to death was defined as the time since the 12- or 24month time point for patients who achieved EFS12/EFS24 and time since the first event for patients who did not achieve EFS12/EFS24. For COD by transformation analysis, which was based only on the US cohort, time to death was defined using the time since the date of transformation in the set of patients with transformation. CODs in patients without transformation were analyzed using the time to death since diagnosis in all patients from diagnosis, with censoring at the date of transformation in the set of patients who experienced transformation before death. All analyses were performed using SAS (SAS/STAT User's Guide, Version 9.4; SAS Institute, Cary, NC) and R (version 3.2.3).

\section{RESULTS}

\section{Descriptive Characteristics}

Baseline characteristics, treatments, and summary outcome data are described for each cohort and the pooled cohort of 1,654 patients (Table 1). French and US cohorts were similar in age structure, sex distribution, Ann Arbor stage, and Follicular Lymphoma International Prognostic Index (FLIPI) score. IC was the most common initial therapy in the French cohort (67\%), whereas in the US cohort IC $(38 \%)$ and watch and wait (36\%) were the most common initial therapies.

In the French cohort, after a median follow-up of 89 months for living patients, there were 113 deaths. In the US cohort, after a median follow-up of 84 months for living patients, there were 170 deaths. Ten-year OS was comparable in the French (79.8\%) and US (76.6\%) cohorts (Appendix Fig A1, online only), as was EFS24 (69.9\% in each cohort; Table 1).

\section{Cumulative Incidence and Distribution of CODs}

We were able to classify COD in 248 (88\%) of 283 decedents. As shown in Figure 1, death as a result of lymphoma was the most common COD, with a cumulative incidence of $10.3 \%$ at 10 years $(95 \% \mathrm{Cl}, 8.6 \%$ to $12.2 \%)$, followed by TRM (3.0\%; $95 \% \mathrm{Cl}, 2.2 \%$ to $4.1 \%$ ), other malignancy $(2.9 \% ; 95 \% \mathrm{Cl}, 2.0 \%$ to $4.2 \%)$, other causes $(2.2 \% ; 95 \% \mathrm{Cl}, 1.5 \%$ to $3.1 \%)$, and unknown cause (3.0\%; $95 \% \mathrm{Cl}, 2.1 \%$ to $4.4 \%$ ). 
TABLE 1. Characteristics of the French, US, and Pooled Cohorts

\begin{tabular}{|c|c|c|c|}
\hline \multirow[b]{2}{*}{ Characteristic } & \multicolumn{3}{|c|}{ Cohort } \\
\hline & $\begin{array}{c}\text { French } \\
(n=734)\end{array}$ & $\begin{array}{c}\text { US } \\
(\mathrm{n}=920)\end{array}$ & $\begin{array}{c}\text { Pooled } \\
(\mathrm{N}=1,654)\end{array}$ \\
\hline \multicolumn{4}{|l|}{ Age at diagnosis, years } \\
\hline Median & 57 & 60 & 59 \\
\hline Range & $(24.0-95.0)$ & $(19.0-93.0)$ & (19.0-95.0) \\
\hline \multicolumn{4}{|l|}{ Sex } \\
\hline Female & 359 (48.9) & 438 (47.6) & $797(48.2)$ \\
\hline Male & $375(51.1)$ & $482(52.4)$ & $857(51.8)$ \\
\hline \multicolumn{4}{|l|}{ Stage } \\
\hline I-II & $178(25.5)$ & 287 (31.8) & $465(29.1)$ \\
\hline III-IV & $520(74.5)$ & $615(68.2)$ & 1135 (70.9) \\
\hline Missing & 36 & 18 & 54 \\
\hline \multicolumn{4}{|l|}{ FLIPI } \\
\hline Low $(0-1)$ & 206 (28.9) & 355 (39.2) & $561(34.7)$ \\
\hline Intermediate (2) & $289(40.5)$ & 309 (34.1) & 598 (36.9) \\
\hline High (3-5) & $218(30.6)$ & $242(26.7)$ & $460(28.4)$ \\
\hline Missing & 21 & 14 & 35 \\
\hline \multicolumn{4}{|l|}{ Initial treatment } \\
\hline Immunochemotherapy & 485 (66.9) & 349 (38.0) & $834(50.7)$ \\
\hline Watch and wait & $132(18.2)$ & 326 (35.5) & 458 (27.9) \\
\hline $\mathrm{R}$ monotherapy & $49(6.8)$ & $111(12.1)$ & $160(9.7)$ \\
\hline Other treatment & $59(8.1)$ & $133(14.5)$ & 192 (11.7) \\
\hline Missing & 9 & 1 & 10 \\
\hline \multicolumn{4}{|l|}{ Death } \\
\hline Yes & $113(15.4)$ & $170(18.5)$ & $283(17.1)$ \\
\hline No & $621(84.6)$ & 750 (81.5) & $1371(82.9)$ \\
\hline \multicolumn{4}{|l|}{ EFS24 } \\
\hline Achieved & 500 (69.9) & 633 (69.9) & 1133 (69.9) \\
\hline Did not achieve & $215(30.1)$ & 272 (30.1) & 487 (30.1) \\
\hline Missing & 19 & 15 & 34 \\
\hline
\end{tabular}

NOTE. Data are given as No. (\%) unless otherwise indicated.

Abbreviations: EFS24, event-free survival at 24 months; FLIPI, Follicular Lymphoma International Prognostic Index; R, rituximab.

The cumulative incidence for each of these causes demonstrated a monotonic (essentially linear) increase over the first decade after diagnosis of FL. Table 2 summarizes the CODs. Among 248 decedents with a known COD, 57\% were a result of lymphoma, $17 \%$ were treatment related, $13 \%$ were a result of other malignancies, and $13 \%$ were a result of other causes (provided in Appendix Table A1, online only). Whereas these distributions were broadly similar in the two cohorts, there was a higher percentage of deaths attributed to lymphoma in the French (65\%) versus the US (50\%) cohort, and a lower percentage of deaths attributed to other causes in the French (7\%) versus the US (18\%) cohort. However, outcomes were similar on the basis of the more valid comparison using cumulative incidence with competing risk of death; the 10-year cumulative incidence of death as a result of lymphoma was 14.2\% $(95 \% \mathrm{Cl}, 11.4 \%$ to $17.7 \%$ ) in the French cohort and $12.5 \%$ $(95 \% \mathrm{Cl}, 10.2 \%$ to $15.4 \%)$ in the US cohort, whereas the 10-year cumulative incidence of death as a result of other causes was 3.7\% (95\% Cl, 2.3\% to 6.1\%) and $6.1 \%(95 \%$ $\mathrm{Cl}, 4.4 \%$ to $8.4 \%$ ), respectively.

Of the 140 deaths as a result of lymphoma, 77 (55\%) occurred in patients whose FL had transformed at some point in the disease history. Of the 42 deaths as a result of TRM, 20 (48\%) were because of infection, 12 (29\%) because of MDS/AML, six (14\%) because of cardiotoxicity, and four ( $9 \%$ ) because of other treatment-related causes.

\section{COD Pattern by Demographic and Clinical Characteristics}

We next modeled the cumulative incidence for each COD category for key subgroups defined on demographic and clinical characteristics. To increase interpretability, we grouped COD as lymphoma-related deaths (lymphoma or treatment related), deaths unrelated to lymphoma (other malignancies and other causes), and deaths as a result of unknown causes. We also estimated the 5- and 10-year cumulative incidence along with 95\% Cls for lymphomarelated and non-lymphoma-related deaths by subgroup and conducted a formal statistical test for differences in cumulative incidence by subgroup (Table 3 ). There was no difference in the cumulative incidence of lymphomarelated $(P=.62)$ or non-lymphoma-related $(P=.20)$ deaths by sex (Appendix Fig A2, online only). As expected, the cumulative incidence of lymphoma-related $(P<.001)$ and non-lymphoma-related $(P<.001)$ deaths increased with age at diagnosis. Of note, in all age groups, the cumulative incidence in lymphoma-related deaths were higher than that of non-lymphoma-related deaths (Fig 2). For example, the 10-year cumulative incidence of lymphoma-related deaths was $9.4 \%$ in patients who were diagnosed at $\geq 60$ years, $14.2 \%$ for patients diagnosed at age 61 to 70 years, and $25.4 \%$ for patients diagnosed at older than age 70 years. The respective 10 -year cumulative incidence of non-lymphoma-related mortality was $1.5 \%$, $5.8 \%$, and $16.6 \%$, respectively.

The cumulative incidence of lymphoma-related mortality increased more rapidly with time since diagnosis in patients with stage III to IV disease compared with stage I to II disease (Appendix Fig A3, online only), with a 10-year cumulative incidence of $15.5 \%$ for stage III to IV compared with $7.8 \%$ for stage I to II disease $(P<.001)$. In contrast, there was no difference in the cumulative incidence of non-lymphoma-related deaths by stage $(P=.58)$. Similarly, the cumulative incidence of lymphoma-related mortality increased more rapidly with a higher FLIPI score (Fig 3), with a 10-year cumulative incidence of $4.0 \%$ for FLIPI 0 to $1,10.0 \%$ for FLIPI 2 , and $27.4 \%$ for FLIPI 3 to $5(P<.001)$, but there was no difference in the cumulative incidence of non-lymphoma-related deaths by FLIPI score $(P=.15)$. Of 


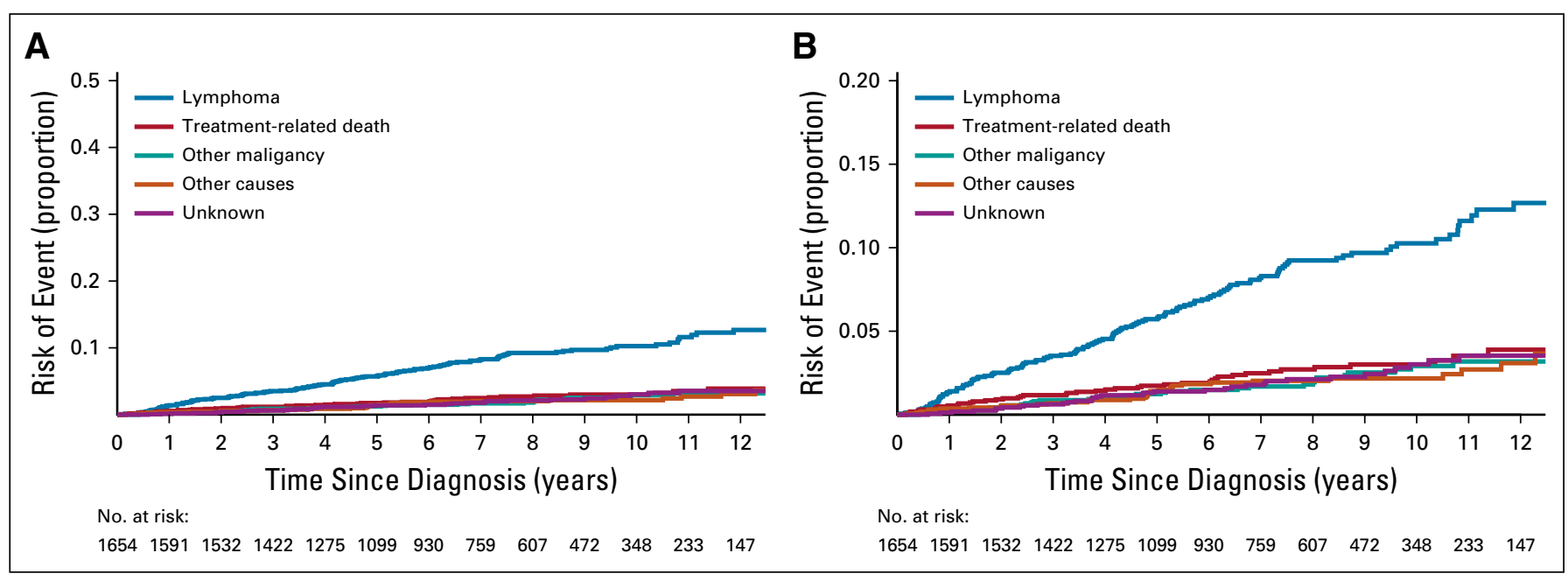

FIG 1. Cumulative incidence for the competing risks of cause of death in the pooled cohort. (A) Cumulative incidence by cause of death. (B) Cumulative incidence by cause of death with $y$-axis rescaled.

note, in patients with FLIPI 0 to 1 disease, the 10-year cumulative incidence of non-lymphoma-related deaths $(3.7 \%)$ was similar to that of lymphoma-related deaths (4.0\%).

\section{COD Pattern by Early Event and Transformation Status}

Among patients who achieved EFS24, the subsequent cumulative incidences of lymphoma- and non-lymphomarelated mortality were similar, with a 10-year cumulative incidence since the EFS24 time point of $6.7 \%$ for lymphoma-related mortality and $5.7 \%$ for non-lymphomarelated mortality (Table 3). In contrast, for patients who did not achieve EFS24, the subsequent cumulative incidence of lymphoma-related mortality was greater than that of

TABLE 2. Causes of Death

\begin{tabular}{llll} 
& \multicolumn{3}{c}{ Cohort } \\
\cline { 2 - 4 } Cause & $\begin{array}{c}\text { French } \\
(\mathbf{n}=\mathbf{1 1 3})\end{array}$ & $\begin{array}{c}\text { US } \\
(\mathbf{n = 1 7 0 )}\end{array}$ & $\begin{array}{c}\text { Pooled } \\
(\mathbf{N}=\mathbf{2 8 3})\end{array}$ \\
\hline Lymphoma & $70(65.4)$ & $70(49.6)$ & $140(56.5)$ \\
\hline Transformed & 42 & 35 & 77 \\
\hline Treatment related & $17(15.9)$ & $25(17.7)$ & $42(16.9)$ \\
\hline MDS/AML & 6 & 6 & 12 \\
\hline Therapy, infection & 6 & 14 & 20 \\
\hline Therapy, cardiac & 2 & 4 & 6 \\
\hline Therapy, other & 3 & 1 & 4 \\
\hline Other cancer & $13(12.1)$ & $20(14.2)$ & $33(13.3)$ \\
\hline Other causes* & $7(6.5)$ & $26(18.4)$ & $33(13.3)$ \\
\hline Missing $\dagger$ & 6 & 29 & 35
\end{tabular}

NOTE. Data are given as No. (\%) unless otherwise indicated.

Abbreviations: AML, acute myeloid leukemia; MDS, myelodysplastic syndrome.

* Other causes listed in Appendix Table A1.

† Missing category is not included in the percentages. non-lymphoma-related mortality, with a 10-year cumulative incidence from an event of $36.1 \%$ for lymphomarelated mortality and $7.0 \%$ for non-lymphoma-related mortality. The higher cumulative incidence of lymphomarelated $(36.1 \% \vee 6.7 \% ; P<.001)$ and non-lymphomarelated $(5.7 \% \vee 7.0 \% ; P=.0052)$ mortality in patients who did not achieve EFS24 compared with patients who achieved EFS24 were both statistically significant. Stratifying the analysis to EFS24 for patients who were initially treated with IC and to EFS12 for patients who were initially treated with all other approaches (non-IC treated), the subsequent cumulative incidence of lymphoma- and non-lymphomarelated mortality were similar (Appendix Fig A4A, online only), with a 10-year cumulative incidence from the EFS24 (IC treated) or EFS12 (non-IC treated) time points of 8.8\% and $8.2 \%$ for lymphoma-related mortality and $4.1 \%$ and $7.1 \%$, respectively, for non-lymphoma-related mortality. In contrast, for patients who did not achieve EFS24 (for IC treated) or EFS12 (for non-IC treated), the subsequent cumulative incidence of lymphoma-related mortality was greater than that of non-lymphoma-related mortality (Appendix Fig A4B), with a 10-year cumulative incidence from an event of $52.4 \%$ and $24.0 \%$ for lymphoma-related mortality and $5.6 \%$ and $9.0 \%$ for non-lymphoma-related mortality, respectively. There were too few events in the non-IC-treated group to provide estimates by specific type of initial management-that is, observation versus rituximab monotherapy versus other.

For the US cohort, we had data on the transformation status for all patients. Among patients without transformation, the cumulative incidence of lymphoma- and non-lymphomarelated mortality were similar (Appendix Fig A5A, online only), with a 10-year cumulative incidence since diagnosis of $8.1 \%$ for lymphoma-related mortality and $6.2 \%$ for non-lymphoma-related mortality. In contrast, the subsequent cumulative incidence of lymphoma-related 


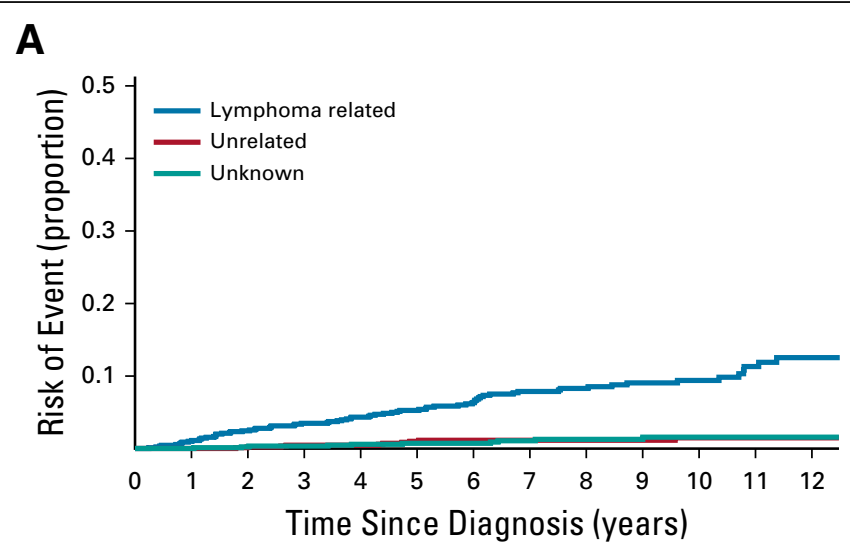

No. at risk:

$\begin{array}{llllllllllllll}\text { age }<60 & 916 & 896 & 864 & 807 & 745 & 647 & 559 & 462 & 377 & 294 & 223 & 151 & 102\end{array}$

\section{C}

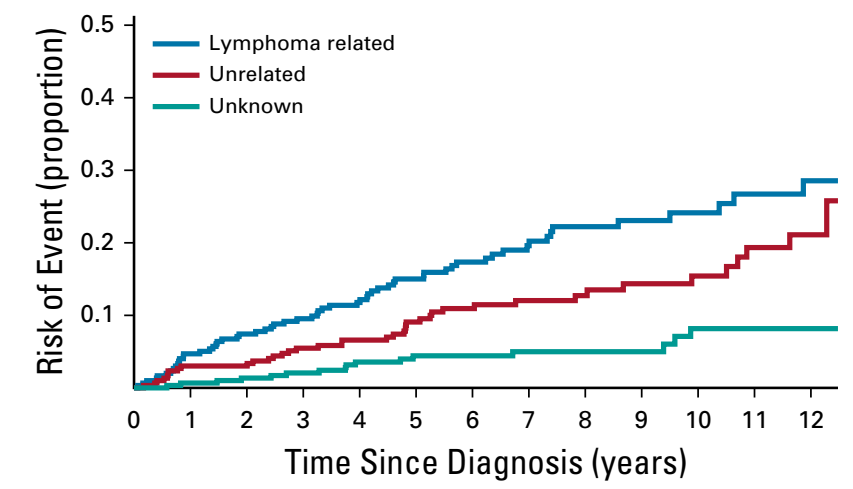

B

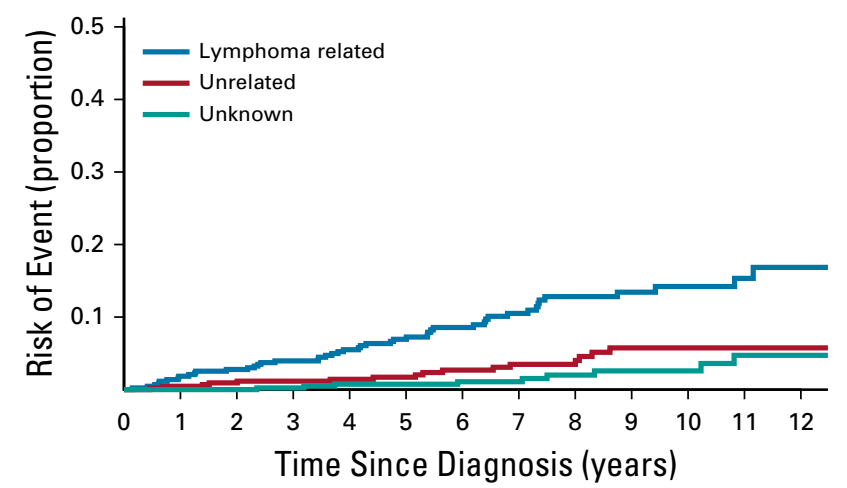

No. at risk:

$\begin{array}{llllllllllllll}\text { age } 60-69 & 435 & 422 & 409 & 384 & 331 & 291 & 242 & 195 & 152 & 116 & 81 & 50 & 31\end{array}$

No. at risk:

$\begin{array}{llllllllllllll}\text { age } 70+ & 300 & 270 & 256 & 228 & 198 & 160 & 128 & 102 & 78 & 62 & 44 & 32 & 14\end{array}$

FIG 2. Cumulative incidence for the competing risks of cause of death. (A) Cumulative incidence by cause of death for patients age $\leq 60$ years. (B) Cumulative incidence by cause of death for patients age 61 to 70 years. (C) Cumulative incidence by cause of death for patients age $>70$ years.

mortality after transformation was greater than that of non-lymphoma-related mortality (Appendix Fig A5B), with a 10-year cumulative incidence since transformation of $45.9 \%$ for lymphoma-related mortality and $4.7 \%$ for non-lymphoma-related mortality.

\section{DISCUSSION}

There are several clinically relevant conclusions from our pooled analysis of two cohort studies of patients with $\mathrm{FL}$ who were diagnosed and treated in the rituximab era. First, OS was approximately $80 \%$ at 10 years since diagnosis in both US and French cohorts, which is comparable to the long-term outcome of patients included in the SWOG ${ }^{12}$ (ClinicalTrials.gov identifier: NCT000006721) or PRIMA (Clinicaltrials.gov identifier: NCT00140582) trials. ${ }^{13}$ Second, lymphoma was the leading $C O D$ in the first decade since diagnosis, with a cumulative risk of mortality as a result of lymphoma of $10.3 \%$ at 10 years, which increased to $13.3 \%$ when combined with TRM. In contrast, a cumulative risk of mortality as a result of non-lymphoma-related causes was only $5.1 \%$ at 10 years. Third, lymphoma-related mortality was the major COD in all age groups, even for patients older than age 70 years. Fourth, whereas FLIPI score was strongly associated with lymphomarelated mortality, it was not associated with non-lymphomarelated mortality. Fifth, the 10-year cumulative risk of lymphoma-related mortality in patients who did not achieve EFS24 was $36.1 \%$. Finally, the cumulative risk of lymphomarelated mortality after transformation was $45.9 \%$.

The strengths of the current study include the use of two independent cohorts, review and classification of all deaths, availability of clinical data, and use of a competing risk analysis to model cumulative incidence. The survival rate in these French and US reference centers were comparable to the OS reported in the literature, ${ }^{7,23}$ which suggests minimal bias and good external validity. However, these cohorts were not population based and future work should evaluate these findings in this setting. A limitation of this real-world study is that management strategies (eg, surveillance, retreatment, and repeat biopsy) were not protocol driven but were at the discretion of the managing physician, and the effect of this on COD is unknown. We also did not have systematic prospective data collection on 


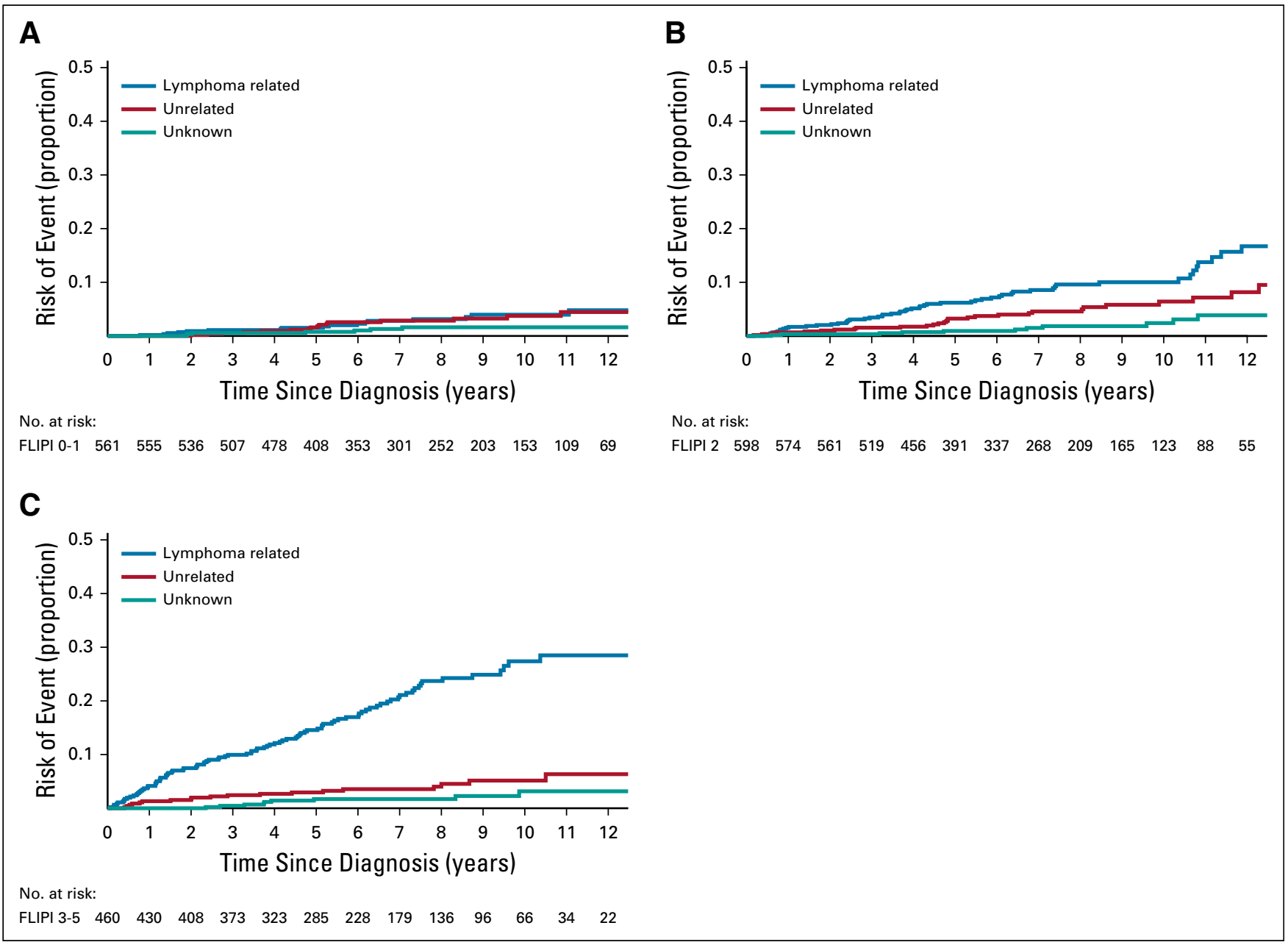

FIG 3. Cumulative incidence for the competing risks of cause of death according to Follicular Lymphoma International Prognostic Index (FLIPI) score. (A) Cumulative incidence by cause of death for patients with FLIPI score 0 to 1. (B) Cumulative incidence by cause of death for patients with FLIPI score 2. (C) Cumulative incidence by cause of death for patients with FLIPI score 3 to 5.

long-term toxicity, which could potentially underestimate TRM. Indeed, is it likely that not all deaths related to chronic toxicities (ie, pulmonary fibrosis from alkylators, or peripheral vascular disease from radiation) were captured. Other limitations include missing data for some deaths and a lack of racial or ethnic diversity. Finally, we only had data on the first decade of deaths in the rituximab era, and longer follow-up is needed to understand late deaths in this cancer with a long natural history.

Whereas recent reports have demonstrated an improvement in excess mortality rate among patients with $\mathrm{FL}$ since the introduction of immunotherapy in combination with chemotherapy, ${ }^{9,24}$ the leading COD remains lymphoma. Indeed, our findings corroborate the long-term follow-up of the younger cohorts of patients with symptomatic disease included in the SWOG trial. ${ }^{12}$ Mounier at al ${ }^{9}$ have reported that the excess mortality rate was higher in the elderly population and in those with stage III to IV disease, which is in agreement with our findings that showed that stage was strongly associated with lymphoma-related death and that lymphoma remained the principal COD in patients older than age 70 years. As expected and reported, ${ }^{25,26}$ we also observed a more important rate of non-lymphoma-related death among patients $\geq 70$ years of age. A recent ${ }^{24}$ population-based study that included 961 patients with FL (with a short 55-month median follow-up) also reported that the higher standardized mortality ratio for $\mathrm{FL}$ (standardized mortality ratio, including all causes of death) was associated with FLIPI score and EFS12/ EFS24 failures. Of note, in the current study, women tended to have a higher standardized mortality ratio than men but a similar global OS in contrast to that reported in the National LymphoCare Study ${ }^{27}$ and the Swedish Lymphoma Registry study $^{28}$, both of which demonstrated lower lymphoma-related mortality for women. ${ }^{27,28}$ In our study, the cumulative incidence of lymphoma-related deaths by sex were identical, whereas a nonsignificant trend toward a lower rate of nonlymphoma-related deaths was observed in women. Of note, of the 140 lymphoma deaths, 77 (55\%) were related to transformation. Indeed, among patients without a transformation during their disease history, the cumulative incidence of death related to lymphoma is equivalent to that for deaths unrelated 
- 这

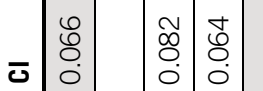

总

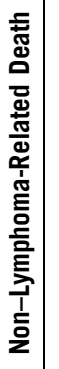

0

(2)

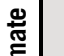

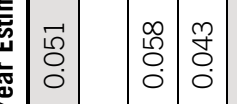

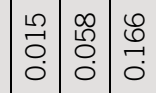

总:

)

융 융

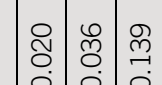

운

$\begin{array}{ll}0 & 0 \\ \infty & 0 \\ 0 & 0 \\ 0 & 0 \\ 0 & 0 \\ 0 & 0\end{array}$

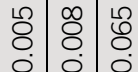

c

这

苞

힝

点

迎

$\frac{8}{\frac{8}{\pi}}$

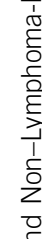

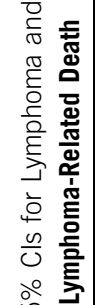

- ङ

ㅎ․

苦

$8 \%$

웡 중

\begin{tabular}{cc|c|c|}
\hline & 0 & 0 & 0 \\
0 & 0 & 0 \\
0 & 0 & 0 & 0 \\
0 & 0 & 0 \\
0 & 0 & 0
\end{tabular}

吕

웅 옹

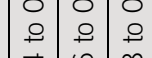

$\begin{array}{lll}0 & 0 \\ 0 & 0 & 0 \\ 0 & 0 & 0 \\ 0 & 0 & 0 \\ 0 & 0 \\ 0\end{array}$

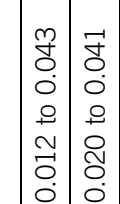

$\begin{array}{lll} & \\ \tilde{N} & \tilde{N} \\ 0 & 0 \\ 0 & 0 \\ 0 & 0 \\ 0 & 0 \\ 0 & 0 \\ 0 & 0 \\ 0 & 0 \\ 0 & 0 \\ 0 & 0\end{array}$

₹ิ

$\begin{array}{ll} & \\ 0 & \tilde{D} \\ 0 & 0 \\ 0 & 0\end{array}$

$\overrightarrow{8}$

槖

峑

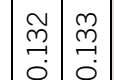

每

愛递

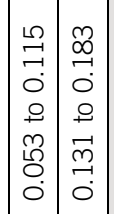

द.

$\overline{8}$

ì

$\overline{0}$

$\infty$

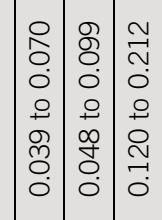

善:

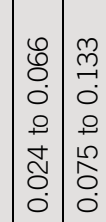

愛

哆

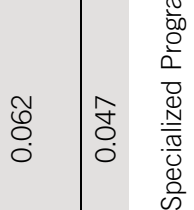

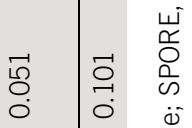

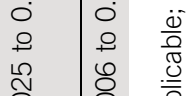

范

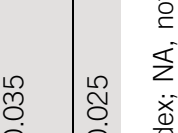

$\therefore:$ :

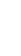

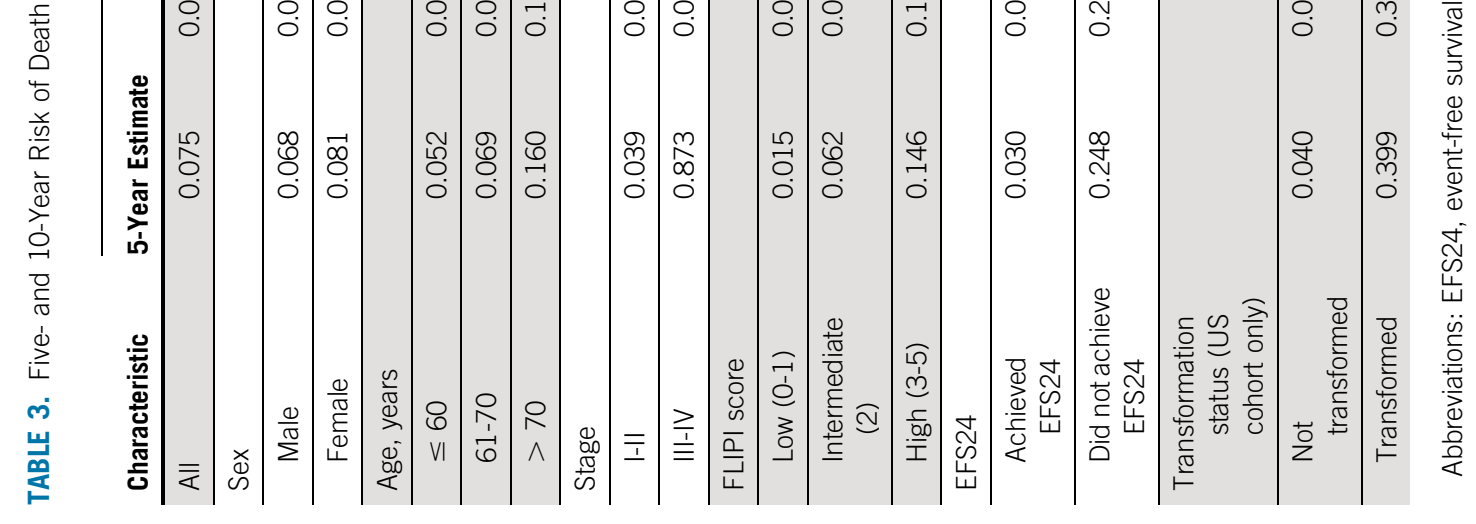


to lymphoma $(4.0 \%$ v3.5\% at 5 years and $8.1 \%$ v $6.2 \%$ at 10 years for deaths related to lymphoma $v$ deaths unrelated to lymphoma, respectively). This important notion that transformation in FL is the major cause of lymphoma-related death should provide a framework for future specific approaches.

In our series, $17 \%$ of deaths $(n=42)$ were attributed to treatment, and these were most commonly a result of infections $(n=20)$ and secondary MDS/AML $(n=12)$. Others have reported a significant incidence of fatal secondary myeloid neoplasia in patients who received radioimmunotherapy or a fludarabine-mitoxantrone-based regimen ${ }^{12}$; however, little is known regarding other long-term, treatment-related fatal complications, such as infections or cardiac deaths, especially in patients with indolent lymphomas. Finally, the risk of nonmyeloid neoplasia (and myeloid secondary neoplasia) in survivors of lymphoma is well known and seems to be higher in patients with FL than in those with diffuse large B-cell lymphoma. ${ }^{29}$ Long-term outcome data of

\section{AFFILIATIONS}

${ }^{1}$ Hospices Civils de Lyon, Centre Hospitalier Lyon Sud; Université de Lyon, Université Claude Bernard Lyon 1, Centre de Recherche en Cancérologie de Lyon INSERM 1052, Lyon, France

${ }^{2}$ Mayo Clinic, Rochester, MN

${ }^{3}$ University of lowa, lowa City, IA

${ }^{4}$ Centre Léon Bérard, Lyon, France

\section{CORRESPONDING AUTHOR}

Gilles Salles, MD, Hospices Civils de Lyon, 165 Chemin du Grand Revoyet, Pierre-Bénite, 69495, Lyon, France; e-mail: gilles.salles@chulyon.fr.

\section{EQUAL CONTRIBUTION}

C.S. and M.J.M. contributed equally to this work. J.R.C. and G.S. jointly supervised this work.

\section{PRIOR PRESENTATION}

Presented at the 34th International Conference on Machine Learning, Sydney, NSW, Australia, August 6-11, 2017. Presented at the International Conference on Malignant Lymphoma, ICML, Lugano, Switzerland, June 14-17, 2017 clinical trials, registry data, and additional studies are needed to have a better estimate of the long-term fatalities directly or indirectly associated with specific treatments.

In conclusion, we provide a comprehensive description of the pattern of deaths observed in patients with $\mathrm{FL}$ in the rituximab era. Despite a favorable (80\%) 10-year OS, lymphoma represents the leading COD in the first decade after diagnosis. This is particularly true for patients who present with a high FLIPI score, for those with transformed disease, and for those who did not achieve EFS12/EFS24. Deaths related to treatment seem to also be a significant burden and new, less-toxic treatment options need to be investigated. Given the adverse outcomes associated with early treatment failure (EFS24) and histologic transformation, efforts that focus on clinical or biologic models to identify at-risk patients and the development of new therapeutic approaches are an important challenge in FL.

\section{SUPPORT}

Supported by National Institutes of Health Grants No. P50-CA97274 and U01-CA195568.

AUTHORS' DISCLOSURES OF POTENTIAL CONFLICTS OF INTEREST AND DATA AVAILABLITY STATEMENT

Disclosures provided by the authors and data availability statement (if applicable) are available with this article at DOI https://doi.org/10.1200/ JC0.18.00400

\section{AUTHOR CONTRIBUTIONS}

Conception and design: Clémentine Sarkozy, Matthew J. Maurer, James R. Cerhan, Gilles A. Salles

Provision of study materials or patients: All authors

Collection and assembly of data: All authors

Data analysis and interpretation: Clémentine Sarkozy, Matthew J. Maurer, James R. Cerhan, Gilles A. Salles, Hervé Ghesquières, Emmanuel Bachy,

Brian K. Link, Thomas M. Habermann

Manuscript writing: All authors

Final approval of manuscript: All authors

Accountable for all aspects of the work: All authors

\section{REFERENCES}

1. Campo E, Swerdlow SH, Harris NL, et al: The 2008 WHO classification of lymphoid neoplasms and beyond: Evolving concepts and practical applications. Blood 117:5019-5032, 2011

2. Teras LR, DeSantis CE, Cerhan JR, et al: 2016 US Iymphoid malignancy statistics by World Health Organization subtypes. CA Cancer J Clin 10.3322/ caac.21357 [epub ahead of print on September 12, 2016]

3. Roulland S, Faroudi M, Mamessier E, et al: Early steps of follicular lymphoma pathogenesis. Adv Immunol 111:1-46, 2011

4. Kridel R, Chan FC, Mottok A, et al: Histological transformation and progression in follicular lymphoma: A clonal evolution study. PLoS Med 13:e1002197, 2016

5. Link BK, Maurer MJ, Nowakowski GS, et al: Rates and outcomes of follicular lymphoma transformation in the immunochemotherapy era: A report from the University of lowa/MayoClinic Specialized Program of Research Excellence Molecular Epidemiology Resource. J Clin Oncol 31:3272-3278, 2013

6. Casulo C, Nastoupil L, Fowler NH, et al: Unmet needs in the first-line treatment of follicular lymphoma. Ann Oncol 28:2094-2106, 2017

7. Wagner-Johnston ND, Link BK, Byrtek M, et al: Outcomes of transformed follicular lymphoma in the modern era: A report from the National LymphoCare Study (NLCS). Blood 126:851-857, 2015

8. Salles G, Ghesquières H: Current and future management of follicular lymphoma. Int J Hematol 96:544-551, 2012

9. Mounier M, Bossard N, Belot A, et al: Trends in excess mortality in follicular lymphoma at a population level. Eur J Haematol 94:120-129, 2015

10. Tan D, Horning SJ, Hoppe RT, et al: Improvements in observed and relative survival in follicular grade 1-2 lymphoma during 4 decades: The Stanford University experience. Blood 122:981-987, 2013 
11. Sant M, Minicozzi P, Mounier M, et al: Survival for haematological malignancies in Europe between 1997 and 2008 by region and age: Results of EUROCARE-5, a population-based study. Lancet Oncol 15:931-942, 2014

12. Shadman M, Li H, Rimsza L, et al: Continued excellent outcomes in previously untreated patients with follicular lymphoma after treatment with CHOP plus rituximab or CHOP plus ${ }^{131}$-tositumomab: Long-term follow-up of phase III randomized study SWOG-S0016. J Clin Oncol 36:697-703, 2018

13. Salles $G$, Seymour JF, Offner $F$, et al: Rituximab maintenance for 2 years in patients with high tumour burden follicular lymphoma responding to rituximab plus chemotherapy (PRIMA): A phase 3, randomised controlled trial. Lancet 377:42-51, 2011

14. Solal-Céligny $\mathrm{P}$, Bellei M, Marcheselli L, et al: Watchful waiting in low-tumor burden follicular lymphoma in the rituximab era: Results of an F2-study database. J Clin Oncol 30:3848-3853, 2012

15. Ardeshna KM, Qian W, Smith P, et al: Rituximab versus a watch-and-wait approach in patients with advanced-stage, asymptomatic, non-bulky follicular lymphoma: An open-label randomised phase 3 trial. Lancet Oncol 15:424-435, 2014

16. Haines IE: Should the paradigm shift for low-grade follicular non-Hodgkin lymphoma be toward less therapy? J Clin Oncol 31:1797-1798, 2013

17. Maurer MJ, Bachy E, Ghesquières $\mathrm{H}$, et al: Early event status informs subsequent outcome in newly diagnosed follicular lymphoma. Am J Hematol 91 : 1096-1101, 2016

18. Casulo C, Byrtek M, Dawson KL, et al: Early relapse of follicular lymphoma after rituximab plus cyclophosphamide, doxorubicin, vincristine, and prednisone defines patients at high risk for death: An analysis from the National LymphoCare study. J Clin Oncol 33:2516-2522, 2015

19. Conconi A, Motta M, Bertoni F, et al: Patterns of survival of follicular lymphomas at a single institution through three decades. Leuk Lymphoma 51:1028-1034, 2010

20. Cerhan JR, Link BK, Habermann TM, et al: Cohort profile: The lymphoma Specialized Program of Research Excellence (SPORE) Molecular Epidemiology Resource (MER) cohort study. Int J Epidemiol 46:1753-1754i, 2017

21. Al-Tourah AJ, Gill KK, Chhanabhai M, et al: Population-based analysis of incidence and outcome of transformed non-Hodgkin's lymphoma. J Clin Oncol 26: 5165-5169, 2008

22. Gray RJ: A class of K-sample tests for comparing the cumulative incidence of a competing risk. Ann Stat 16:1141-1154, 1988

23. Provencio M, Sabín P, Gomez-Codina J, et al: Impact of treatment in long-term survival patients with follicular lymphoma: A Spanish Lymphoma Oncology Group registry. PLoS One 12:e0177204, 2017

24. Provencio M, Royuela A, Torrente M, et al: Prognostic value of event-free survival at 12 and 24 months and long-term mortality for non-Hodgkin follicular lymphoma patients: A study report from the Spanish Lymphoma Oncology Group. Cancer 123:3709-3716, 2017

25. Maartense E, Le Cessie S, Kluin-Nelemans HC, et al: Age-related differences among patients with follicular lymphoma and the importance of prognostic scoring systems: Analysis from a population-based non-Hodgkin's lymphoma registry. Ann Oncol 13:1275-1284, 2002

26. Alig S, Jurinovic V, Pastore A, et al: Impact of age on genetics and treatment efficacy in follicular lymphoma. Haematologica 103:e364-e367, 2018

27. Nabhan C, Zhou X, Day BM, et al: Disease, treatment, and outcome differences between men and women with follicular lymphoma in the United States. Am J Hematol 91:770-775, 2016

28. Junlén HR, Peterson S, Kimby E, et al: Follicular lymphoma in Sweden: Nationwide improved survival in the rituximab era, particularly in elderly women—A Swedish Lymphoma Registry study. Leukemia 29:668-676, 2015

29. Morton LM, Curtis RE, Linet MS, et al: Second malignancy risks after non-Hodgkin's lymphoma and chronic lymphocytic leukemia: Differences by lymphoma subtype. J Clin Oncol 28:4935-4944, 2010

\section{Listen to the New ASCO University Podcasts - New Content Weekly}

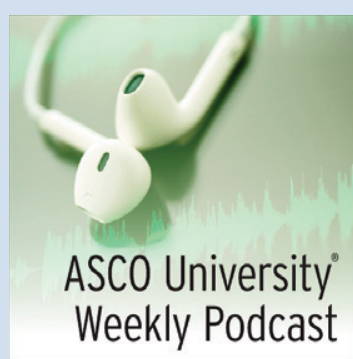

ASCO University Podcasts episodes feature insightful and informative content centered around self-assessment questions, ASCO guidelines, and new drugs in oncology. Available exclusively through an ASCO University Essentials subscription and to Fellows enrolled in EEOF.

Listen now at university.asco.org/podcasts 
Cause of Death in Follicular Lymphoma in the First Decade of the Rituximab Era: A Pooled Analysis of French and US Cohorts

The following represents disclosure information provided by authors of this manuscript. All relationships are considered compensated. Relationships are self-held unless noted. I = Immediate Family Member, Inst = My Institution. Relationships may not relate to the subject matter of this manuscript. For more information about ASCO's conflict of interest policy, please refer to www.asco.org/rwc or ascopubs.org/jco/site/ifc.

\section{Clémentine Sarkozy}

Honoraria: Genentech, Celgene

Research Funding: Genentech (Inst), Takeda

Travel, Accommodations, Expenses: Takeda

Matthew J. Maurer

Consulting or Advisory Role: MorphoSys

Research Funding: Kite Pharma (Inst), Celgene (Inst), NanoString Technologies (Inst)

\section{Brian K. Link}

Consulting or Advisory Role: Genentech, AbbVie, Gilead Sciences, Celgene Research Funding: Genentech (Inst), Pharmacyclics (Inst), Janssen

Pharmaceuticals (Inst)

Travel, Accommodations, Expenses: Genentech, Celgene

Hervé Ghesquieres

Honoraria: Gilead Sciences

Consulting or Advisory Role: Gilead Sciences, Celgene

Travel, Accommodations, Expenses: Gilead Sciences, Amgen, Roche

\section{Andrew L. Feldman}

Consulting or Advisory Role: Infinity Pharmaceuticals

Patents, Royalties, Other Intellectual Property: Inventor of technology for which Mayo Clinic holds an unlicensed patent or has submitted a patent application (Inst)
Stephen M. Ansell

Honoraria: WebMD, Research to Practice

Research Funding: Bristol-Myers Squibb (Inst), Seattle Genetics (Inst), Affirmed Therapeutics (Inst), Regeneron (Inst), Pfizer (Inst), LAM Therapeutics (Inst), Trillium Therapeutics (Inst)

Emmanuel Bachy

Honoraria: Gilead Sciences, Roche, Amgen, Janssen-Cilag

Consulting or Advisory Role: Roche

Travel, Accommodations, Expenses: Janssen-Cilag

James R. Cerhan

Consulting or Advisory Role: Janssen Pharmaceuticals Research Funding: NanoString Technologies, Celgene

\section{Gilles Salles}

Honoraria: Genentech, Amgen, Janssen Pharmaceuticals, Bristol-Myers Squibb, Celgene, Servier, Gilead Sciences, Novartis

Consulting or Advisory Role: Genentech, Gilead Sciences, Janssen

Pharmaceuticals, Celgene, Novartis, Merck, Novimmune, Pfizer, Acerta Pharma, Kite Pharma, Bristol-Myers Squibb, Servier

Research Funding: Genentech (Inst), Gilead Sciences, Celgene, Genentech, Merck

Travel, Accommodations, Expenses: Genentech, Sanofi

No other potential conflicts of interest were reported. 


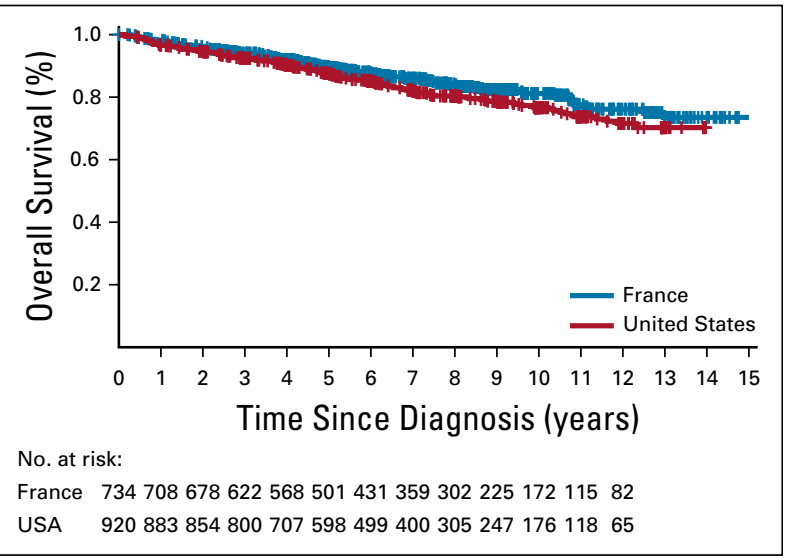

FIG A1. Overall survival since diagnosis.

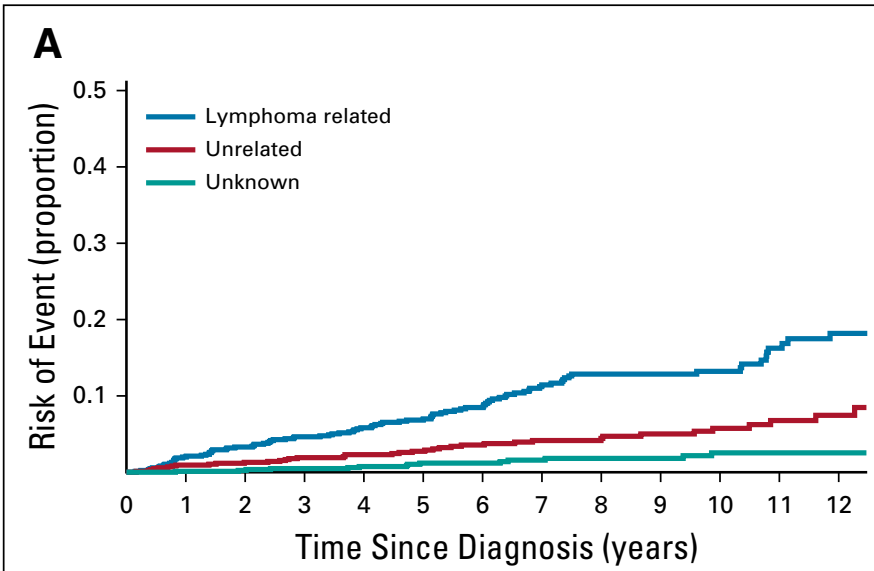

\section{B}

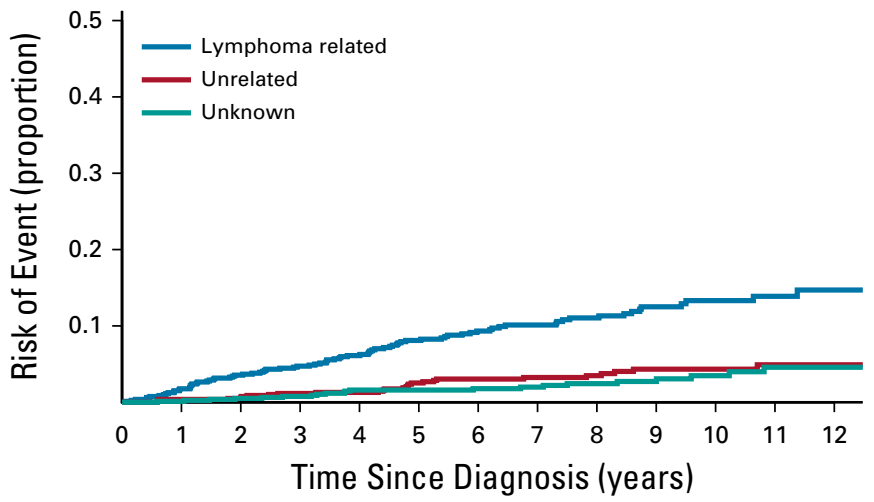

No. at risk:

No. at risk:

$\begin{array}{llllllllllllll}\text { female } & 797 & 770 & 741 & 687 & 614 & 532 & 457 & 387 & 311 & 233 & 160 & 110 & 65\end{array}$

FIG A2. Cumulative incidence for the competing risks of cause of death according to sex. (A) Cumulative incidence by cause of death for male patients. (B) Cumulative incidence by cause of death for female patients. 


\section{A}

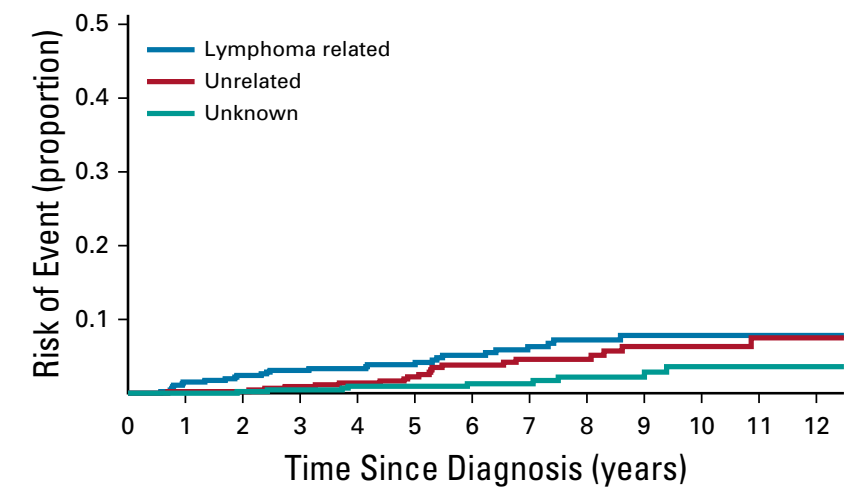

No. at risk:

$\begin{array}{llllllllllllll}\text { Stage I-II } & 465 & 451 & 432 & 402 & 364 & 305 & 250 & 202 & 161 & 123 & 87 & 60 & 38\end{array}$
B

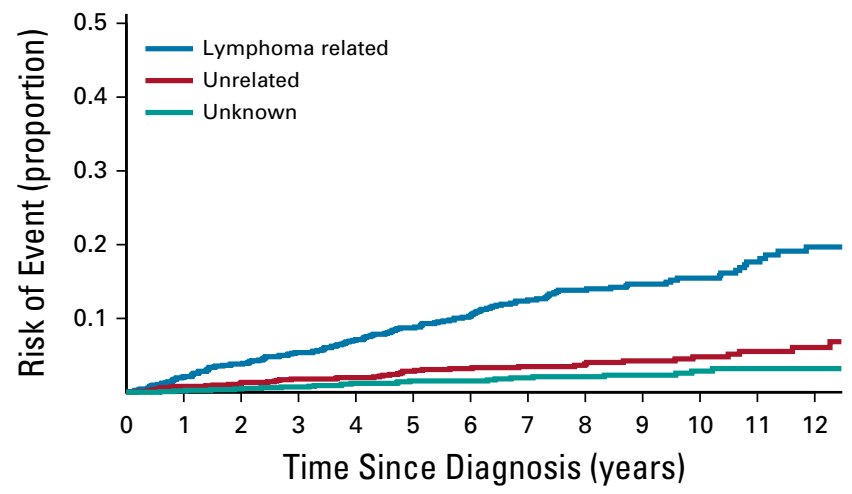

No. at risk:

Stage III-IV $1135 \quad 1088 \quad 1051 \quad 978 \quad 874 \quad 762 \quad 651 \quad 532 \quad 422 \quad 328 \quad 243 \quad 163 \quad 102$

FIG A3. Cumulative incidence for the competing risks of cause of death according to disease stage. (A) Cumulative incidence by cause of death for patients with stage I and II disease. (B) Cumulative incidence by cause of death for patients with stage III and IV disease.

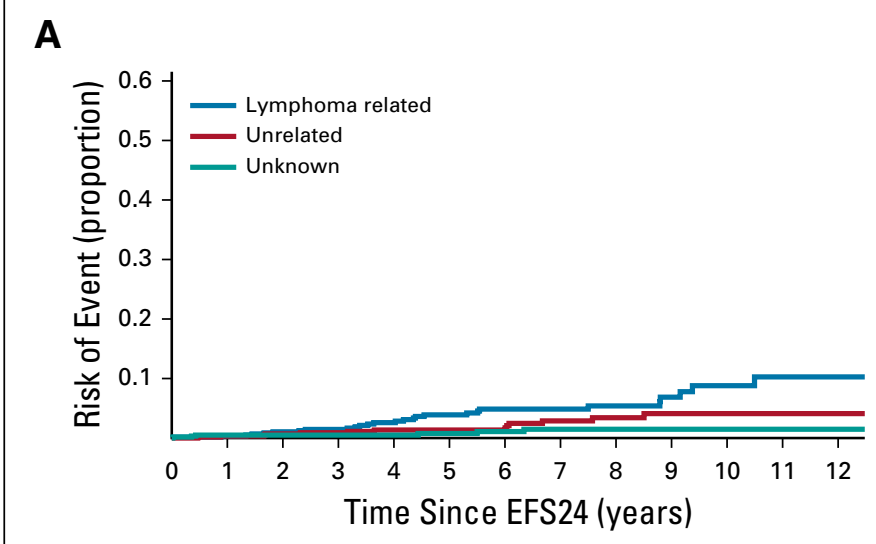

No. at risk:

$\begin{array}{lllllllllllllll}\text { IC EFS24 } & 611 & 608 & 607 & 572 & 513 & 448 & 384 & 312 & 252 & 193 & 148 & 101 & 66\end{array}$

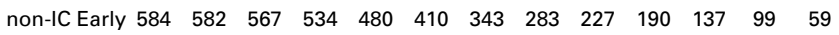

C

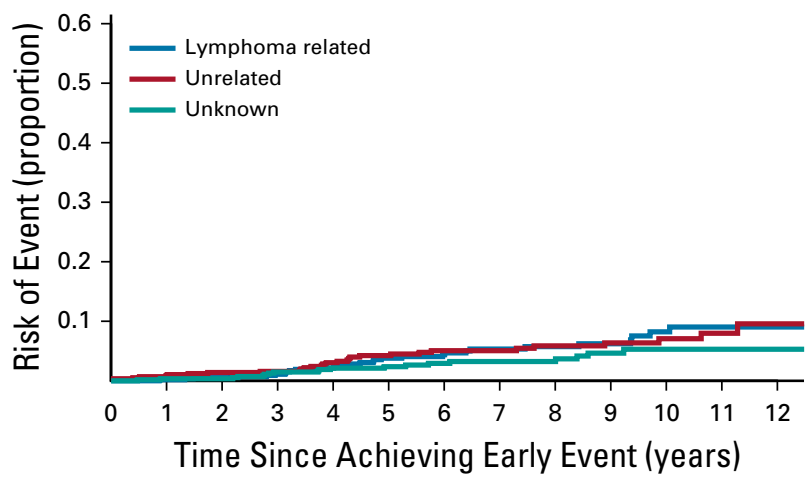

B

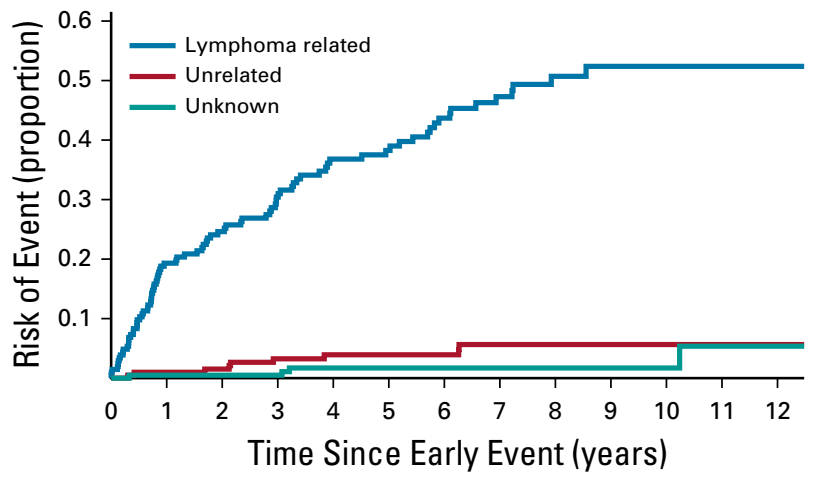

No. at risk:

$\begin{array}{llllllllllllll}\text { IC fail EFS24 } & 216 & 188 & 160 & 132 & 108 & 89 & 77 & 58 & 45 & 32 & 20 & 10 & 7\end{array}$

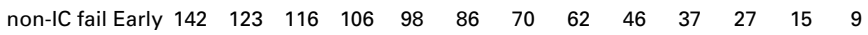

D

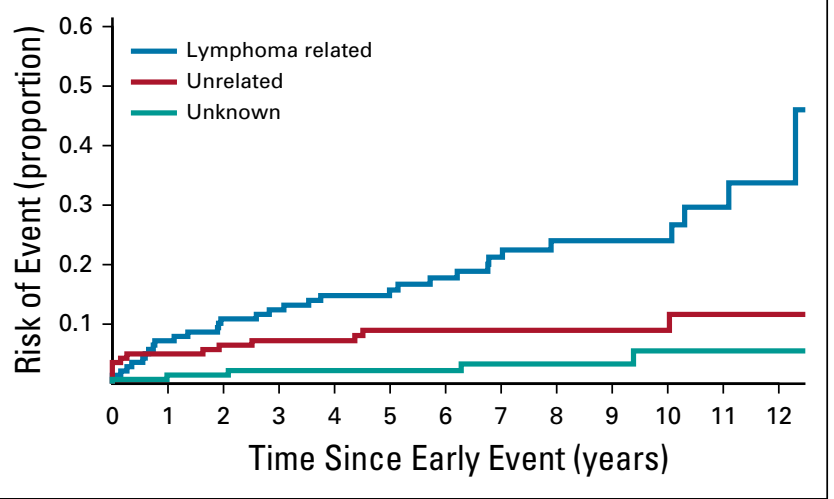

FIG A4. Cumulative incidence for the competing risks of cause of death. (A) Patients treated upfront with immunochemotherapy (IC) who achieved event-free survival within 24 months of diagnosis (EFS24). (B) Patients treated upfront with IC who did not achieve EFS24 diagnosis. (C) Patients not treated with up-front IC (non-IC treated) who achieved EFS12/early event achieve non-IC treated. (D) Patients not treated with up-front IC (non-IC treated) who did not achieve EFS12 (early event)/early event fail non-IC treated. 
A

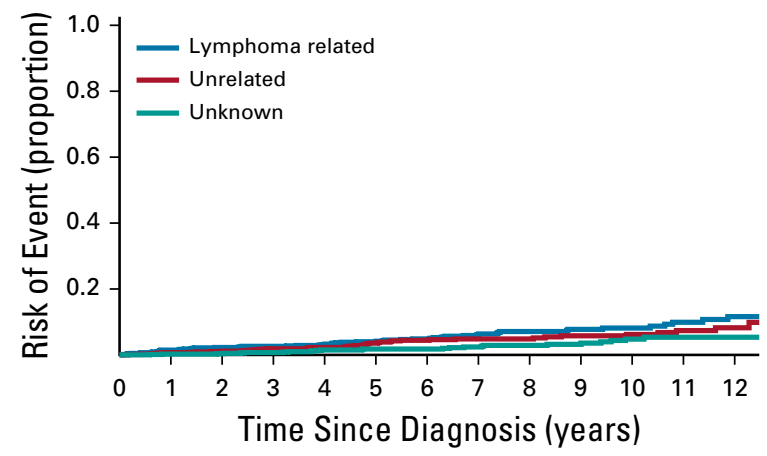

No. at risk:

no transformation $920 \quad 883 \quad 854 \quad 800 \quad 707 \quad 598 \quad 499 \quad 400 \quad 305 \quad 247 \quad 176 \quad 118 \quad 65$
B

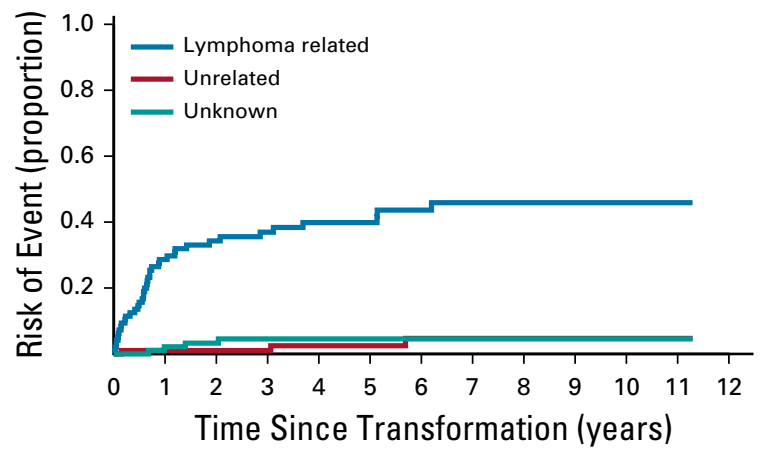

No. at risk:

$\begin{array}{llllllllllllll}\text { transformation } & 97 & 87 & 80 & 72 & 63 & 54 & 47 & 36 & 26 & 22 & 19 & 14 & 9\end{array}$

FIG A5. Cumulative incidence for the competing risks of cause of death. (A) US cohort patients without follicular lymphoma transformation/US cohort no transformation (B) US cohort patients with follicular lymphoma transformation/US cohort transformation. 
TABLE A1. Description of Other Causes of Death

Center

Other Cause of Death

\begin{tabular}{|c|c|}
\hline CHLS & Cardiorespiratory arrest for food misuse (no lymphoma) \\
\hline CHLS & Sudden death (no lymphoma) \\
\hline CHLS & Cholecystisis (no lymphoma) \\
\hline CHLS & Cardiac decompensation (no lymphoma) \\
\hline CHLS & Denutrition (no lymphoma) \\
\hline CLB & CR at last follow-up, dead at age 92 years \\
\hline CLB & Epilepsia-vascular accident-stable disease \\
\hline SPORE & Peripheral vascular disease, unspecified \\
\hline SPORE & Parkinson disease \\
\hline SPORE & Necrotizing fasciitis \\
\hline SPORE & Sepsis \\
\hline SPORE & Atherosclerotic heart disease \\
\hline SPORE & Stroke \\
\hline SPORE & Acute myocardial infarction \\
\hline SPORE & Subarachnoid hemorrhage \\
\hline SPORE & Acute ischemia of the bowel \\
\hline SPORE & Acute myocardial infarction \\
\hline SPORE & Pneumonia \\
\hline SPORE & Acute respiratory distress syndrome \\
\hline SPORE & Diffuse lung injury \\
\hline SPORE & Pneumonia/sever sepsis \\
\hline SPORE & Congestive heart failure \\
\hline SPORE & Vascular disease \\
\hline SPORE & Pneumonia \\
\hline SPORE & Acute myocardial infarction \\
\hline SPORE & Ruptured aortic aneurysm \\
\hline SPORE & $\begin{array}{l}\text { Occlusion and stenosis of precerebral arteries, not } \\
\text { resulting in cerebral infarction }\end{array}$ \\
\hline SPORE & Natural causes, stated on death certificate \\
\hline SPORE & Acute cardiopulmonary arrest \\
\hline SPORE & Acute myocardial infarction \\
\hline SPORE & $\begin{array}{l}\text { Other causes, not related to lymphoma, treatment, or } \\
\text { cancer }\end{array}$ \\
\hline SPORE & Cardiac arrest \\
\hline SPORE & $\begin{array}{l}\text { Other causes, not related to lymphoma, treatment, or } \\
\text { cancer }\end{array}$ \\
\hline
\end{tabular}

Abbreviations: CHLS, Centre Hospitalier Lyon Sud; CLB, Centre Léon Bérard; CR, complete response; SPORE, Specialized Program of Research Excellence. 